\title{
Higgs lepton flavor violation
}

\author{
Juan Herrero-Garcia* \\ Department of Theoretical Physics, Royal Institute of Stockholm (KTH) \\ E-mail: juhg@kth.se
}

\begin{abstract}
We study lepton-flavor-violating Higgs decays in the light of the recently observed deviation in the $H \rightarrow \tau \mu$ channel with respect to the Standard Model, which should be confirmed/excluded in the second run of the LHC. Starting from an EFT approach, we discuss both tree-level and looplevel UV completions. In particular, we find that, in agreement with previous analyses, loop-level realizations are in general too suppressed, while models containing at least two Higgs doublets can explain the enhancement. We also analyze well-motivated neutrino mass models that could in principle accommodate the excess, finding two popular examples: the Zee model and left-right symmetric models.
\end{abstract}

The European Physical Society Conference on High Energy Physics 22-29 July 2015

Vienna, Austria

${ }^{*}$ Speaker. 


\section{Motivation: Higgs lepton flavor violation as a signal of new physics}

Lepton flavor violation (LFV) has been observed in neutrino oscillations. In principle, there is no fundamental reason not to expect it in the charged lepton sector as well (cLFV), in processes like $\tau \rightarrow \mu \gamma$ and $\mu \rightarrow 3 e$, or in the Higgs sector in processes like $H \rightarrow \tau \mu$ (HLFV). However, when one computes the different light neutrino contributions to these processes one finds out that in general they are completely unobservable. Therefore, any excess will immediately point-out to physics beyond the SM, and beyond the light neutrino contribution. Furthermore, generically an observed signal in any of these processes will point to a mechanism for neutrino masses distinct from the simplest high-scale $\mathrm{SO}(10)$-motivated seesaw scenario, and, if the signal is indeed related to Majorana neutrino masses, it would teach us about the UV completion of the Weinberg operator.

In this work we study HLFV from an effective field theory (EFT) approach, explicitly listing all the topologies of the UV realizations. Furthermore, we discuss the connection of HLFV to neutrino masses [1]. One of the main motivations for the study is the recent hint of an HLFV excess observed by CMS [2]. It is a $2.4 \sigma$ deviation from the SM prediction:

$$
B R(H \rightarrow \mu \tau)=\left(0.84_{-0.37}^{+0.39}\right) \%
$$

while ATLAS shows no significant deviation [3], with only a small excess in one of the signal regions that is not statistically significant.

As in the SM the Higgs couplings are diagonal in the lepton mass basis, new physics is needed. Parameterizing the violation of flavor by $y_{\mathrm{ij}}$, the Lagrangian reads:

$$
\mathscr{L}=-\overline{e_{\mathrm{Li}}} m_{\mathrm{i}} e_{\mathrm{Ri}}-H \overline{e_{\mathrm{Li}}} y_{\mathrm{ij}} e_{\mathrm{Rj}}+\text { H.c. },
$$

where $\mathrm{H}$ is the Higgs boson. Therefore, the predicted branching ratio of $H \rightarrow \tau \mu$ is:

$$
\mathrm{BR}(H \rightarrow \tau \mu)=\frac{m_{H}}{8 \pi \Gamma_{H}^{\mathrm{total}}}\left(\left|y_{\tau \mu}\right|^{2}+\left|y_{\mu \tau}\right|^{2}\right),
$$

where the total width $\Gamma_{H}^{\mathrm{total}}$ encodes the SM width and the new contribution, i.e., $\Gamma_{H}^{\mathrm{total}}=\Gamma_{H}^{\mathrm{SM}}+$ $\Gamma_{H}^{\text {new }}{ }^{1}$ It is easy to check that, in order to explain the $\sim 1 \%$ excess, we need:

$$
0.002 \lesssim \sqrt{\left|y_{\tau \mu}\right|^{2}+\left|y_{\mu \tau}\right|^{2}} \lesssim 0.003
$$

at $\sim 1 \sigma$. This range is consistent with having hierarchical charged-lepton masses, i.e., $\left|y_{\tau \mu} y_{\mu \tau}\right| \lesssim$ $m_{\mu} m_{\tau} / v^{2} \sim 4 \cdot 10^{-6}$ without fine-tuning. The observed value and the different EFT constraints are summarized in figure 1 of the CMS collaboration [2].

\section{An effective approach}

We will start by using an EFT approach, assuming that the physics responsible for the $H \rightarrow \mu \tau$ excess is above the EW scale. The SM Yukawa Lagrangian reads:

$$
\mathscr{L}_{\mathrm{SM}}=\bar{L} i \not D L+\overline{e_{\mathrm{R}}} i \not D e_{\mathrm{R}}+\bar{L} Y_{\mathrm{e}} e_{\mathrm{R}} \Phi+\text { H.c. },
$$

\footnotetext{
${ }^{1} \mathrm{Using} \mathrm{BR}(H \rightarrow \tau \tau)=0.065$, one can estimate $\mathrm{BR}(H \rightarrow \tau \mu) \simeq 0.065\left(\left|y_{\tau \mu}\right|^{2}+\left|y_{\mu \tau}\right|^{2}\right) /\left(2\left|y_{\tau \tau}\right|^{2}\right)$.
} 


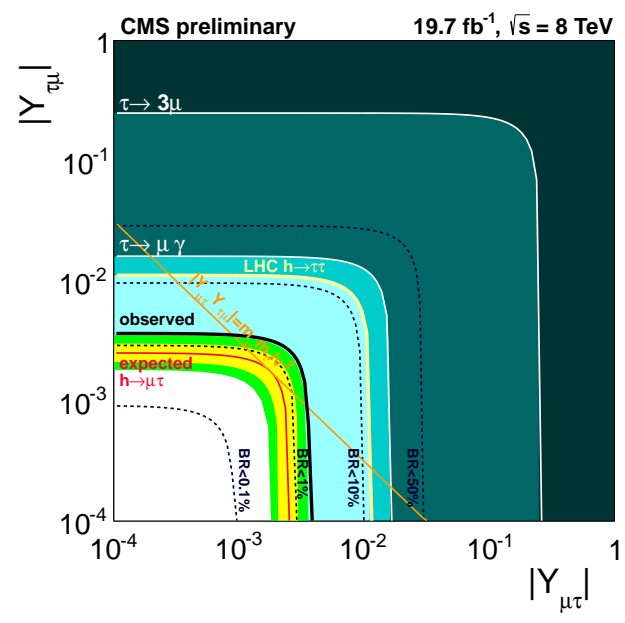

Figure 1: CMS observed value and cLFV constraints on the $y_{\tau \mu}-y_{\mu \tau}$ plane [2].

where $\Phi$ SM Higgs doublet and $L\left(e_{\mathrm{R}}\right)$ the lepton doublets (singlets). $Y_{\mathrm{e}}$ is a general Yukawa matrix, which one can take to be diagonal without loss of generality.

At the non-renormalizable level there are operators that generate HLFV. This is the case of the Yukawa operator (see for instance [4, 5, 6]), which reads:

$$
\mathscr{L}_{\mathrm{HLFV}}^{D=6}=\frac{1}{\Lambda^{2}} \bar{L} C e_{\mathrm{R}} \Phi\left(\Phi^{\dagger} \Phi\right)+\text { H.c. },
$$

where $C$ is a general matrix in flavor space.

After SSB, $\left\langle\Phi_{0}\right\rangle=(H+v) / \sqrt{2}$, we need to diagonalize the charged lepton mass matrix, $M_{e}$ :

$$
D_{\mathrm{e}} \equiv \operatorname{diag}\left(m_{e}, m_{\mu}, m_{\tau}\right)=\frac{1}{\sqrt{2}} V_{\mathrm{L}}^{\dagger}\left(Y_{\mathrm{e}}+C \frac{v^{2}}{2 \Lambda^{2}}\right) V_{\mathrm{R}} v .
$$

In the mass basis the Yukawas are no longer diagonal and they give rise to Higgs decays that violate flavor (renaming $V_{\mathrm{L}}^{\dagger} C V_{\mathrm{R}} \rightarrow C$ ), with effective Yukawas:

$$
\left(Y_{\mathrm{e}}\right)_{i j}=\frac{m_{i} \sqrt{2}}{v} \delta_{i j}+C_{i j} \frac{v^{2}}{\Lambda^{2}} .
$$

One can open the Yukawa operator in order to see which UV completions can generate it. The different topologies are shown in figure 2 and listed in table 1 . There are topologies with only scalars:

- A: A type-III 2HDM where both Higgses take a VEV can explain the excess [7, 8, 9]. In this case HLFV is given by:

$$
B R(H \rightarrow \mu \tau)=\frac{m_{H}}{8 \pi \Gamma_{H}}\left(\frac{Y_{2}^{\tau \mu} s_{\beta-\alpha}}{\sqrt{2} s_{\beta}}\right)^{2},
$$

where $\tan \beta=v_{1} / v_{2}$ and $\alpha$ the CP-neutral scalars mixing angle. We will give two wellmotivated examples, which are also well-known neutrino mass models. 


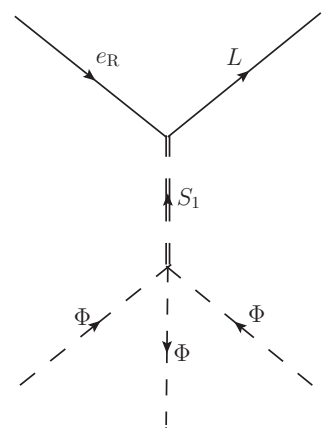

(A)

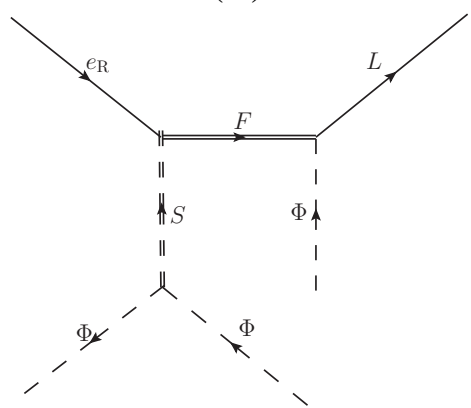

(C)



(B)

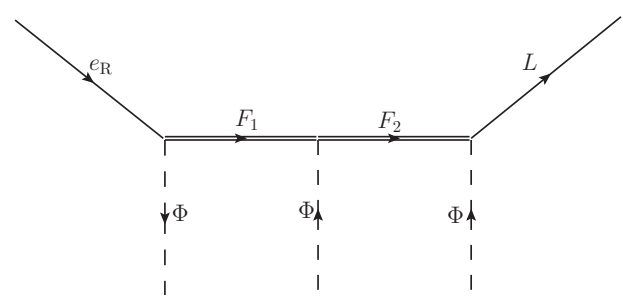

(D)

Figure 2: Tree-level topologies of the Yukawa operator. The $\Phi$ and the $\bar{\Phi}$ can be interchanged among the different legs. See table 1 for the different representations.

- B: While the $Y=0$ singlet is less constrained, the triplets have strong bounds from the $\rho$ parameter, which implies that $\mu_{2} v^{2} /\left(v M_{S_{2}}^{2}\right) \lesssim(5 \mathrm{GeV}) / v^{2}$ Thus:

$$
B R(H \rightarrow \mu \tau) \simeq 0.065\left(Y_{S_{1}} \frac{\mu_{1} v}{M_{S_{1}}^{2}} \frac{\mu_{2} v^{2}}{v M_{S_{2}}^{2}} \frac{1}{y_{\tau}}\right)^{2} \lesssim 0.6 \frac{Y_{S_{1}}^{2} v^{2}}{M_{S_{1}}^{2}}
$$

Moreover, in general it is expected than whenever topology B is present, topology also A is, and it will be dominant.

There are also topologies with both scalars and vector-like (VL) fermions (C), and with only VL fermions (D) [9, 10], see table 1. All the explicit UV completions with VL fermions, however, also generate the Derivative operators shown in table 2 and depicted in figure 3 . They are related by EOM to the Yukawa operator, but the key point we want to emphasize is that the bounds on both are different, as they give rise to different processes. Of course, one can choose the operator basis that is more convenient (as long as it is complete) to check the viability of a model, and the most conservative option is to choose the one that gives the strongest constraints. For HLFV, the Derivative operator generated by VL leptons generates both tree-level FCNC and cLFV at one loop $(\tau \rightarrow \mu \gamma)$, which implies that VL leptons cannot generate a large enough HLFV $[9,10,1]$.

\footnotetext{
${ }^{2}$ The vevs $v_{T}$ of the scalar triplets $(3,0)_{S}$ and $(3,1)_{S}$ contribute as: $\rho_{(3,0)}=1+4 v_{T}^{2} / v^{2}$ and $\rho_{(3,1)}=\left(v^{2}+2 v_{T}^{2}\right) /\left(v^{2}+\right.$ $\left.4 v_{T}^{2}\right)$, respectively. Therefore $(3,0)_{S}$ gives rise to $\rho>1$, while $(3,1)_{S}$ yields $\rho<1$. Notice that experimentally $\rho=$ $1.00040 \pm 0.00024$.
} 


\begin{tabular}{|c|c|c|c|}
\hline Top. & Particles & Representations $\left(\mathrm{SU}(2)_{\mathrm{L}}, \mathrm{U}(1)_{\mathrm{Y}}\right)$ & $\mathrm{H} e_{\alpha} e_{\beta}$ \\
\hline \hline $\mathbf{A}$ & $1 \mathrm{~S}$ & $S=(2,-1 / 2)$ & $\frac{Y \lambda}{m_{S_{1}}^{2}}$ \\
\hline $\mathbf{B}$ & $2 \mathrm{~S}$ & $(2,-1 / 2)_{S} \oplus(1,0)_{S},(3,0)_{S},(3,1)_{S}$ & $\frac{Y \mu_{1} \mu_{2}}{m_{S_{1}} m_{S_{2}}}$ \\
\hline $\mathbf{C}_{\mathbf{1}}$ & $1 \mathrm{~F}, 1 \mathrm{~S}$ & $(2,-1 / 2)_{F} \oplus(1,0)_{S},(3,0)_{S}$ & $\frac{Y_{L} Y_{e} \mu}{m_{F} m_{S}^{2}}$ \\
$\mathbf{C}_{\mathbf{2}}$ & $1 \mathrm{~F}, 1 \mathrm{~S}$ & $(2,-3 / 2)_{F} \oplus(3,1)_{S}$ & $\frac{Y_{L} Y_{e} \mu}{m_{F} m_{S}^{2}}$ \\
$\mathbf{C}_{\mathbf{3}}$ & $1 \mathrm{~F}, 1 \mathrm{~S}$ & $(1,-1)_{F} \oplus(1,0)_{S},(3,-1)_{F} \oplus(3,0)_{S}$ & $\frac{Y_{L} Y_{e} \mu}{m_{F} m_{S}^{2}}$ \\
$\mathbf{C}_{\mathbf{4}}$ & $1 \mathrm{~F}, 1 \mathrm{~S}$ & $(3,0)_{F} \oplus(3,1)_{S}$ & $\frac{Y_{L} Y_{e} \mu}{m_{F} m_{S}^{2}}$ \\
\hline $\mathbf{D}_{\mathbf{1}}$ & $2 \mathrm{~F}$ & $(2,-1 / 2)_{F} \oplus(1,0)_{F},(3,0)_{F}$ & $\frac{Y_{L} Y_{e} Y_{F}}{m_{F} m_{F_{2}}}$ \\
$\mathbf{D}_{\mathbf{2}}$ & $2 \mathrm{~F}$ & $(2,-1 / 2)_{F} \oplus(1,-1)_{F},(3,-1)_{F}$ & $\frac{Y_{L} Y_{e} Y_{F}}{m_{F} m_{F_{2}}}$ \\
$\mathbf{D}_{\mathbf{3}}$ & $2 \mathrm{~F}$ & $(2,-3 / 2)_{F} \oplus(1,-1)_{F},(3,-1)_{F}$ & $\frac{Y_{L} Y_{e} Y_{F}}{m_{F_{1}} m_{F_{2}}}$ \\
\hline
\end{tabular}

Table 1: Tree-level topologies of the Yukawa operator.

\begin{tabular}{|c|c|c|c|c|c|c|}
\hline Operator & Top. & Particles & $\mathrm{Z} v_{\alpha} v_{\beta}$ & $\mathrm{Z} e_{\alpha} e_{\beta}$ & $\mathrm{Wev}$ & $\mathrm{H} e_{\alpha} e_{\beta}$ \\
\hline \hline$\left(\overline{e_{\mathrm{R}}} \Phi^{\dagger}\right) \gamma_{\mu} D^{\mu}\left(e_{\mathrm{R}} \Phi\right)$ & $E_{1}$ & $(2,-1 / 2)_{F}$ & & -1 & & $\frac{Y Y y_{\tau} v^{2}}{m_{F}^{2}}$ \\
\hline$\left(\overline{e_{\mathrm{R}}} \Phi^{T}\right) \gamma_{\mu} D^{\mu}\left(e_{\mathrm{R}} \Phi^{*}\right)$ & $E_{2}$ & $(2,-3 / 2)_{F}$ & & +1 & & $\frac{Y Y y_{\tau} v^{2}}{m_{F}^{2}}$ \\
\hline \hline$(\bar{L} \tilde{\Phi}) \gamma_{\mu} D^{\mu}\left(\tilde{\Phi}^{\dagger} L\right)$ & $E_{3 a}$ & $(1,0)_{F}$ & -1 & & -1 & No \\
\hline$(\bar{L} \tilde{\tau} \tilde{\Phi}) \gamma_{\mu} D^{\mu}\left(\tilde{\Phi}^{\dagger} \vec{\tau} L\right)$ & $E_{3 b}$ & $(3,0)_{F}$ & -1 & -2 & +1 & $\frac{Y Y y_{\tau} v^{2}}{m_{F}^{2}}$ \\
\hline \hline$(\bar{L} \Phi) \gamma_{\mu} D^{\mu}\left(\Phi^{\dagger} L\right)$ & $E_{4 a}$ & $(1,-1)_{F}$ & & +1 & -1 & $\frac{Y_{\mu} Y_{\tau} y_{\tau} \nu^{2}}{m_{F}^{2}}$ \\
\hline$(\bar{L} \vec{\tau} \Phi) \gamma_{\mu} D^{\mu}\left(\Phi^{\dagger} \vec{\tau} L\right)$ & $E_{4 b}$ & $(3,-1)_{F}$ & +2 & +1 & +1 & $\frac{Y Y y_{\tau} v^{2}}{m_{F}^{2}}$ \\
\hline
\end{tabular}

Table 2: Tree-level topologies of the Derivative operator. The gauge boson interactions are given by $Z$ : $c e /\left(2 c_{W} s_{W}\right)$ and $W: c e /\left(2 \sqrt{2} s_{W}\right)$.

The difference between models with VL leptons and 2HDM ones is that in the latter $\tau \rightarrow \mu \gamma$ is suppressed due to the needed chirality flip in the tau lepton, ${ }^{3}$ while in the former chirality can be flipped in the heavy VL lepton, yielding an enhanced rate.
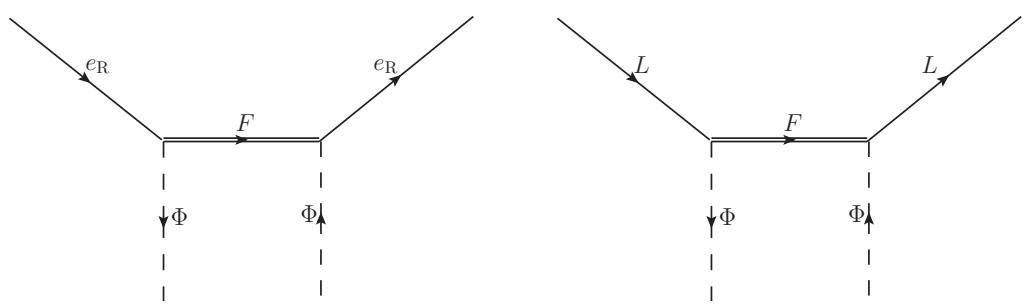

Figure 3: Tree-level topologies of the Derivative operator. See table 2 for the different representations.

\section{Connection to neutrino masses}

As already discussed in the introduction, lepton flavor is violated in the neutral neutrino sector. This poses the question if an observed violation in the charged lepton sector could be somehow related to neutrino masses. In the following we will discuss some of the neutrino mass models and their connection to violation of flavor in Higgs decays.

\footnotetext{
${ }^{3}$ At two loops there is no need to have a chirality flip in the tau lepton. Anyway, the argument is still valid, as these are suppressed with respect to the one-loop contribution to cLFV of VL leptons.
} 

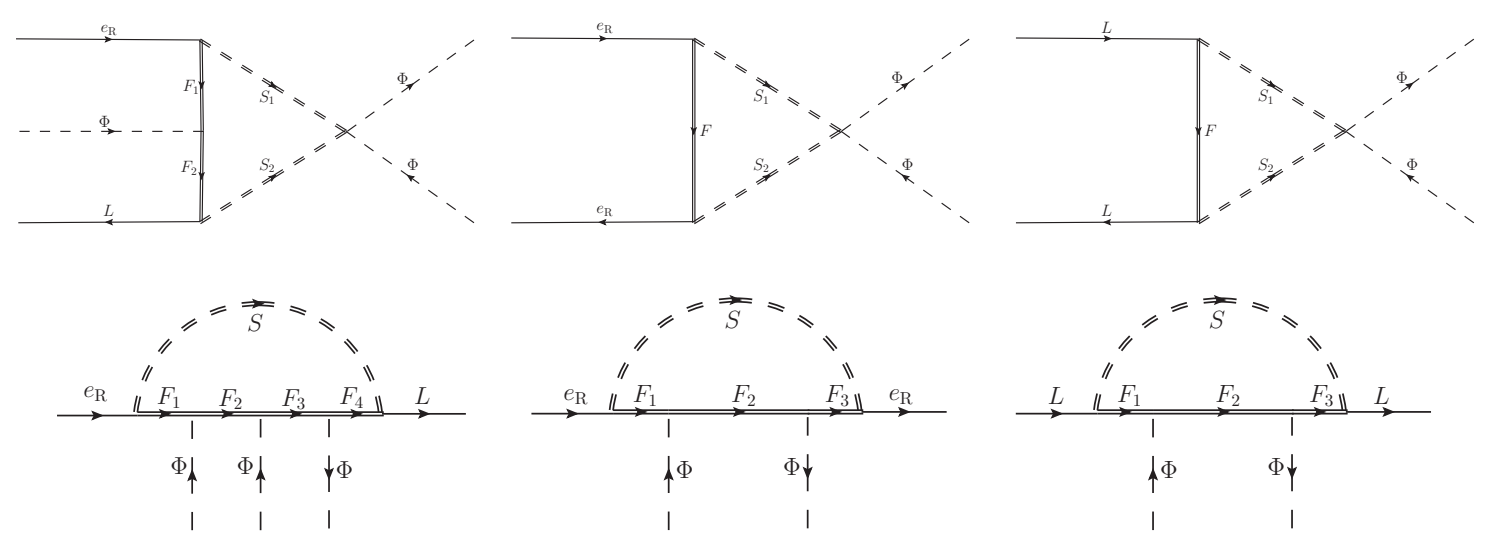

Figure 4: One-loop level topologies giving rise to the Yukawa and the Derivative operators. Some neutrino mass models generate HLFV via this type of diagrams.

\begin{tabular}{|c|c|c|c|}
\hline Top. & Part. & Representations & Neutrino mass models \\
\hline \hline LR & S, F & $(1,0)_{F},(3,0)_{F}$ & Dirac, SSI/III (ISS) \\
\hline RR & S & $(1,2)_{S}$ & ZB (doubly-charged) \\
\hline LL & S & $(1,1)_{S},(3,1)_{S}$ & ZB (singly-charged), SSII \\
\hline \hline LL $\left(Z_{2}\right)$ & $S \oplus F$ & $(1,1 / 2)_{S} \oplus(1,0)_{F},(3,0)_{F}$ & Scotogenic Model \\
\hline
\end{tabular}

\subsection{HLFV at one loop}

There are neutrino mass models that generate HLFV at one loop, as shown in table ?? and depicted in figure 4. In general, these are however suppressed, see for instance ref. [11] where seesaw scenarios were studied. We estimate that most neutrino mass models such as the Zee-Babu model or the Scotogenic model give:

$$
B R(H \rightarrow \mu \tau) \sim 0.06 \frac{\lambda^{2}}{(4 \pi)^{4}}\left(\frac{v}{\mathrm{TeV}}\right)^{4}\left(\frac{Y}{M_{i} / \mathrm{TeV}}\right)^{4},
$$

while $\tau \rightarrow \mu \gamma$ typically imposes the strong constraint:

$$
\left(\frac{Y}{M_{i} / \mathrm{TeV}}\right)^{4} \lesssim \mathscr{O}(0.01-1)
$$

Therefore, the $H \rightarrow \mu \tau$ generated is in general too small, $B R(H \rightarrow \mu \tau) \lesssim 10^{-9}$. One could think whether cLFV constraints can be evaded, but this is not the natural expectation, as some of the new particles of the model are electrically charged and therefore one expects cLFV at the same loop-level as HLFV. If one imposes large Yukawas with special textures one can get $B R$ up to $\lesssim 10^{-5}$, like in inverse seesaw frameworks [12]. But one should keep in mind that large $Y, \lambda$ lead to instabilities/non-perturbative couplings close to the EW scale, and could also generate $H \rightarrow \gamma \gamma$ signals above the current limits depending on the model. Another viable but fine-tuned option is to assume that different contributions to cLFV cancel among themselves. For all these reasons we therefore conclude that it is not very natural to consider models that generate sizable HLFV at one loop (although we emphasize once more that it could be achieved by special textures, large Yukawa couplings or fine-tuned cancellations), and we focus on tree-level realizations. 


\subsection{HLFV at tree level}

\subsubsection{The Zee model}

We have found two very well-motivated models that give rise to both (possibly large) HLFV and neutrino masses. The first one is the Zee Model [13]. It introduces an extra Higgs doublet $\Phi_{2}$ and a singly-charged singlet $h^{+}$. In order to explain neutrino mixings correctly it is necessary that both scalar doublets couple to the charged leptons, and thus it is a type-III 2HDM:

$$
\mathscr{L}_{Y}=-\bar{L}\left(Y_{1} \Phi+Y_{2} \Phi_{2}\right) e_{\mathrm{R}}-\overline{\tilde{L}} f L h^{+}+\text {H.c. }
$$

In this model neutrino masses are generated at one loop:

$$
M_{v} \propto f m_{f}^{2}+m_{f}^{2} f^{T}-v / \sqrt{2 c_{\beta}}\left(f m_{f} Y_{2}+Y_{2}^{T} m_{f} f^{T}\right),
$$

and HLFV is also given by eq. (2.5). Moreover, in order to explain neutrino mixing angles, nontrivial constraints on the Yukawas relevant for HLFV are present. As in all 2HDM, there are strong constraints coming from cLFV, and therefore a detailed study of the model and its constraints is needed in order to see if one can achieve a sizable signal (in preparation [14]).

\subsubsection{Left-right symmetric models}

The second framework which we believe is extremely well-motivated are left-right symmetric models. These are based on the gauge group $\mathrm{SU}(2)_{\mathrm{L}} \times \mathrm{SU}(2)_{\mathrm{R}} \times \mathrm{U}(1)_{\mathrm{B}-\mathrm{L}}$, which restores parity at a high scale:

$$
\mathrm{Q}=\mathrm{T}_{3 \mathrm{~L}}+\mathrm{T}_{3 \mathrm{R}}+\frac{\mathrm{B}-\mathrm{L}}{2} .
$$

In the simplest models two $(\mathrm{B}-\mathrm{L})=2$ triplets, one $\Delta_{\mathrm{R}}(1,3,2)$, one $\Delta_{\mathrm{L}}(3,1,2)$, and one bi-doublet $(2,2,0)$ are added:

$$
\Sigma=\left(\begin{array}{cc}
\Phi_{1}^{0} & \Phi_{2}^{+} \\
\Phi_{1}^{-} & \Phi_{2}^{0}
\end{array}\right), \quad \tilde{\Sigma}=\tau_{2} \Sigma^{*} \tau_{2}=\left(\begin{array}{cc}
\Phi_{2}^{0 *} & -\Phi_{1}^{+} \\
-\Phi_{2}^{-} & \Phi_{1}^{0 *}
\end{array}\right) .
$$

The yukawas read:

$$
\mathscr{L}_{Y}=-\bar{L}_{L}\left(Y_{1} \Sigma+Y_{2} \tilde{\Sigma}\right) L_{R}+\text { H.c. }
$$

and they give rise to a type-III $2 \mathrm{HDM}$ at low energies:

$$
\mathscr{L}_{Y}=\frac{\overline{e_{L}}}{\sqrt{2}}\left(Y_{1}\left(v_{1}+H_{1}^{0}\right)+Y_{2}\left(v_{2}+H_{2}^{0}\right)\right) e_{\mathrm{R}}+\text { H.c. },
$$

which generates HLFV as in eq. (2.5). Notice that in general LR symmetric models one typically needs a certain vev hierarchy, $v_{\mathrm{L}} \ll v_{1} \sim v_{2} \ll v_{\mathrm{R}}$, with $v_{\mathrm{L}}\left(v_{\mathrm{R}}\right)$ the vev of $\Delta_{\mathrm{L}}\left(\Delta_{\mathrm{R}}\right)$.

Important constraints come from FCNC, $m_{H_{2}^{0}} \gtrsim 15 \mathrm{TeV}$. Interestingly, LR models are currently receiving a lot of attention, as they can accommodate a $W_{R}$ boson of $\sim 2 \mathrm{TeV}$ that could in principle explain the recently observed diboson anomaly $[15,16,17]$. Although it seems plausible, whether a LR model could consistently explain both signals deserves further investigation. 


\section{Acknowledgments}

I am grateful to Arcadi Santamaria and Nuria Rius for their collaboration in this work and for many fruitful discussions and comments.

\section{References}

[1] J. Herrero-Garcia, N. Rius, and A. Santamaria, Higgs lepton flavor violation: Uv completions and connection to neutrino masses, In preparation.

[2] CMS Collaboration, V. Khachatryan et al., Search for Lepton-Flavour-Violating Decays of the Higgs Boson, Phys. Lett. B749 (2015) 337-362, [arXiv: 1502 . 07400 ].

[3] ATLAS Collaboration, G. Aad et al., Search for lepton-flavour-violating $H \rightarrow \mu \tau$ decays of the Higgs boson with the ATLAS detector, arXiv: 1508.03372.

[4] A. Goudelis, O. Lebedev, and J.-h. Park, Higgs-induced lepton flavor violation, Phys.Lett. B707 (2012) 369-374, [arXiv:1111.1715].

[5] G. Blankenburg, J. Ellis, and G. Isidori, Flavour-changing decays of a 125 gev higgs-like particle, arXiv: 1202.5704 .

[6] R. Harnik, J. Kopp, and J. Zupan, Flavor violating higgs decays, a rXiv:1209.1397.

[7] S. Davidson and G. Grenier, Lepton flavour violating higgs and tau to mu gamma, arXiv:1001.0434.

[8] D. A. Sierra and A. Vicente, Explaining the cms higgs flavor violating decay excess, arXiv:1409.7690.

[9] I. Doršner, S. Fajfer, A. Greljo, J. F. Kamenik, N. Košnik, and I. Nišandžic, New Physics Models Facing Lepton Flavor Violating Higgs Decays at the Percent Level, JHEP 06 (2015) 108, [arXiv:1502.07784].

[10] A. Falkowski, D. M. Straub, and A. Vicente, Vector-like leptons: Higgs decays and collider phenomenology, arXiv:1312.5329.

[11] E. Arganda, A. M. Curiel, M. J. Herrero, and D. Temes, Lepton flavor violating Higgs boson decays from massive seesaw neutrinos, Phys. Rev. D71 (2005) 035011, [hep-ph/ 0407302 ].

[12] E. Arganda, M. J. Herrero, X. Marcano, and C. Weiland, Imprints of massive inverse seesaw model neutrinos in lepton flavor violating Higgs boson decays, Phys. Rev. D91 (2015), no. 1015001 , [arXiv:1405.4300].

[13] A. Zee, A Theory of Lepton Number Violation, Neutrino Majorana Mass, and Oscillation, Phys. Lett. B93 (1980) 389. [Erratum: Phys. Lett.B95,461(1980)].

[14] In preparation.

[15] ATLAS Collaboration, G. Aad et al., Search for high-mass diboson resonances with boson-tagged jets in proton-proton collisions at $\sqrt{s}=8 \mathrm{TeV}$ with the ATLAS detector, arXiv : 1506.00962.

[16] CMS Collaboration, V. Khachatryan et al., Search for massive resonances decaying into pairs of boosted bosons in semi-leptonic final states at $\sqrt{s}=8 \mathrm{TeV}$, JHEP 08 (2014) 174, [arXiv:1405.3447].

[17] P. S. Bhupal Dev and R. N. Mohapatra, Unified explanation of the eej j, diboson and dijet resonances at the LHC, Phys. Rev. Lett. 115 (2015), no. 18 181803, [arXiv: 1508.02277 ]. 